Cite as: Stephane H Maes, (2020), "Right-handed neutrinos? Mass? Ask Gravity”, viXra:2007.0018v1, https://vixra.org/pdf/2007.0018v1.pdf, https://shmaesphysics.wordpress.com/2020/06/21/right-handedneutrinos-ask-gravity/, June 23, 2020.

\title{
Right-handed neutrinos? Mass? Ask Gravity
}

\author{
Stephane H. Maes ${ }^{1}$
}

June 21, 2020 (V2: 10/11/2020)

\begin{abstract}
:
In a multi-fold universe, gravity emerges from Entanglement through the multi-fold mechanisms. As a result, gravity-like effects appear in between entangled particles that they be real or virtual. Long range, massless gravity results from entanglement of massless virtual particles. Entanglement of massive virtual particles leads to massive gravity contributions at very smalls scales. Multi-folds mechanisms also result into a spacetime that is discrete, with a random walk fractal structure and non-commutative geometry that is Lorentz invariant and where spacetime nodes and particles can be modeled with microscopic black holes. All these recover General relativity at large scales and semi-classical model remain valid till smaller scale than usually expected. Gravity can therefore be added to the Standard Model. This can contribute to resolving several open issues with the Standard Model. In particular with chirality flips of fermion induced by gravity, right-handed neutrinos (and left-handed anti-neutrinos) can appear in flight and now acquire mass when encountering Higgs bosons; two mysteries can be explained in one shot in a multi-fold universe.
\end{abstract}

\section{Introduction}

The new preprint [1] proposes contributions to several open problems in physics like the reconciliation of General Relativity (GR) with Quantum Physics, explaining the origin of gravity proposed as emerging from quantum (EPREinstein Podolsky Rosen) entanglement between particles, detailing contributions to dark matter and dark energy and explaining other Standard Model mysteries without requiring New Physics beyond the Standard Model other than the addition of gravity to the Standard Model Lagrangian. All this is achieved in a multi-fold universe that may well model our real universe, which remains to be validated.

With the proposed model of [1], spacetime and Physics are modeled from Planck scales to quantum and macroscopic scales and semi classical approaches appear valid till very small scales. In [1], it is argued that spacetime is discrete, with a random walk-based fractal structure, fractional and noncommutative at, and above, Planck scales (with a 2-D behavior and Lorentz invariance preserved by random walks till the early moments of the universe). Spacetime results from past random walks of particles. Spacetime locations and particles can be modeled as microscopic black holes (Schwarzschild for photons and spacetime coordinates, and metrics between Reisner Nordstrom [2] and Kerr Newman [3] for massive and possibly charged particles - the latter being possibly extremal). Although surprising, [1] recovers results consistent with other like [4], while also being able to justify the initial assumptions of black holes from the gravity or entanglement model. The resulting gravity model recovers General Relativity at larger scale, as a 4-D process, with massless gravity, but also with massive gravity components at very small scale that make gravity significant these scales. Semi-classical models also work well till way smaller scales that usually expected.

In this paper, we remain at a high level of discussion of the analysis and references are generic for the subjects. It makes the points accessible to a wider audience and keeps the door open to further papers or discussions devoted to details of interest. Yet, it requires the reader to review [1], as we do not revisit here all the details of the multi-

\footnotetext{
${ }^{1}$ shmaes.physics@gmail.com
} 
fold mechanism or reconstruction of spacetime. More targeted references for all the material discussed here are compiled in [1].

\section{2. $\mathrm{SM}_{\mathrm{G}}$ : The Standard Model with Multi-Fold Gravity}

[1] proposes that in a multi-fold universe, the Lagrangian is complemented by terms associated to gravity and entanglement (in the form of the sum of the attractive effective potentials) [1].

$$
\mathscr{L}_{\text {total }}=\mathscr{L}_{S M}+\mathscr{L}_{\text {Gravity-with }-M F}+\mathscr{L}_{\text {EPR }}+\mathscr{L}_{\text {Others } \ldots}
$$

The effect of gravity can be seen through the attractive potential contributions of all the energy sources. It can also been seen as expressing the Standard Model Lagrangian in curved spacetime (semi-classical point of view), now considered valid till small scales.

EPR entanglement is not believed to often play a significant role, except in dark matter use cases [5].

The last term is all other "New Physics" terms and we will consider it to be null.

\section{Chirality and Helicity flips induced by Gravity}

In a curved spacetime, the chirality (or helicity) of massless fermion flips back and forth [6].

In the presence of gravity (with perturbative graviton models), the chirality of massive fermions flips [7]. Additional torsion further contribute to such flips [8]. [1] generates torsion within matter due to the effect of uncertainty on the multi-folds.

These effects have already been analyzed as the reason why gravity can smear the anomalies of baryon and lepton number symmetries and therefore potentially ensure the absence of proton decay, except possibly in extreme conditions within black holes [9].

Note that in the literature, it is also argued that chirality would not flip for massless fermion [7], at the difference of [6]. We believe that the latter is more correct as [7] relies on linearization of gravity, a process that does not work well and that is not giving a correct analysis compared to how gravity is explained in [1] and we know that gravity is not weak any more at very smalls scales, especially due to the massive gravity contributions.

\section{The Right-handed Neutrino and its Left-handed anti particles}

We recommend the following reference as entry point to neutrinos [10].

Neutrinos exist with different flavors and oscillate in flight between these flavors to change flavor and masses. They always interact in a specific flavor with the corresponding mass. Only left-handed neutrinos and right-handed anti neutrinos seem to interact (i.e. when not in flight).

So far, only left-handed neutrinos and right-handed anti-neutrinos have been observed. It is unknown if what happens or happened to the particles with opposite chirality. Do they exist? 


\section{The Neutrino mass problem}

As a result of the absence of these opposite chirality neutrinos cannot interact with the Higgs Boson (which flips chirality). Therefore it is known in the standard model how neutrino have acquired their observed mass (that is now established to by non-zero).

Many theories have been proposed, usually with New Physics, to try to explain the mass. None have been validated so far. They involve hypothesis of seesaw mechanism, Majorana neutrinos (i.e. neutrino as its own self anti-particle), an additional sterile neutrino and a whole bunch of super partners proposals. An overview can be found in [11].

Numerous experiments have been proposed to try to validate on model or another with for example the search for Neutrino-less Double Beta-Decay (e.g. to determine if neutrinos are Majorana particles). It is fair to say that nothing conclusive has been observed so far!

At the light of [1], the reasoning above for $\mathrm{SM}_{\mathrm{G}}$, and [9], we suspect that all these efforts may be going in the wrong direction...

Indeed, if we consider that gravity is present, then in flight particles can flip chirality in addition to oscillating in mass and flavors. So Higgs interactions are now possible in flight, which is how and when bumps with Higgs boson take place -a different situation form particle to particle interactions (also flipping chiralities, to be then flip back to observable chiralities by gravity, before any of all the other types of interaction can take place), which means mass acquisition as conventionally understood for fermions in the Standard Model can occur in flight for neutrinos (also why it is inflight that we can find the neutrino mass eigenstates). Masses are small because available interaction time with Higgs bosons is small.

It resolves in one shot both the questions of the existence of right-handed neutrinos (and left-handed antineutrinos) as well as the origin of the (low) mass of the neutrinos. All is achieved within the context of the Standard Model with Gravity, in a multi-fold universe, and without the need of New Physics.

Also, this analysis is for a Multi-fold universe as in [1]. [1] details arguments and ways to check its relationship with the real universe. Besides properties that can be experimentally verified (in the future because of the macroscopic weakness of gravity and gravity like effects for entangled systems), [1] shows how the multi-fold mechanisms and behaviors are in many aspects in today's conventional physics, that, at times, anticipates the behaviors modeled of a multi-fold universe. In addition, [1] explains many results obtained in gravity, quantum mechanics, General Relativity, superstring theory, Loop Quantum Gravity and the AdS/CFT correspondence conjecture. All these works attempt to come up with models for the real universe. It is at least a good sign that [1] may provide an interesting model of the real universe.

Other theories showing that gravity is relevant at the level of the standard model, can repeat the chirality flip argument, even with no relation to multi-fold universe and mechanisms or to gravity emergence from entanglement. So our model here is generic: if we add gravity to Standard Model with a model keeping it non negligible at the Standard Model scales, then right-handed neutrinos and left-handed anti neutrinos exist in flight, only left-handed neutrinos and right-handed anti neutrinos interact in general; but the existence of both chirality in flight ensures mass acquisition via the Higgs mechanism.

Note however that If our model here is not validated by experience, it would not invalidate the multi-fold mechanism and the proposal that gravity emerges from entanglement as detailed in [1]. The analysis builds on [1], as a consequence of it, but it is not a condition for validation of multi-fold universes. 


\section{Conclusions}

We believe that [1] makes a compelling case for the consistency of its multi-fold proposal. The present paper shows how the mechanisms of multi-fold universes can help address the challenges of explaining the mass of the neutrinos without New Physics.

We explain the fate of right-handed neutrinos and left-handed anti neutrinos: they exist, but only in flight where they can interact with the Higgs. Why it only exist in flight is still an open issue. And the low mass of the neutrinos results from the usual Higgs mechanism, while in flight. The mass is low because only little time is available for mass acquisition and bumping with Higgs bosons). The model works for multi-fold universe as well as in any situation where gravity is non negligible and added to the Standard Model.

This along with similar results in [1] and [9], make a strong case for more seriously considering the implications of adding gravity to the Standard Model to obtain $\mathrm{SM}_{\mathrm{G}}$, as a way to contribute to addressing open issues and offer better alternatives to New Physics speculations. This goes hand in hand with recognizing that this also implies the need to seriously consider that gravity may not always be negligible at the Standard Model scales as proposed in [1].

References: (most references come from popular science to make the discussion more approachable) [1]: Stephane H. Maes, (2020) "Quantum Gravity Emergence from Entanglement in a Multi-Fold Universe", viXra:2006.0088v1, (June 9, 2020).

[2]: https://en.wikipedia.org/wiki/Reissner\%E2\%80\%93Nordstr\%C3\%B6m metric

[3]: https://en.wikipedia.org/wiki/Kerr-Newman metric

[4]: Burinskii, Alexander, (2008), "The Dirac-Kerr-Newman electron", arXiv:0507109v4

[5]: Stephane H Maes, (2020), "Explaining Dark Matter Without New Physics?", viXra:2006.0261v1, https://shmaesphysics.wordpress.com/2020/06/21/explaining-dark-matter-without-new-physics/, June 21, 2020. [6]: Carlos Mergulhao Jr., (1995), "Neutrino Helicity Flip in a Curved Space-tlme", General Relativity and Gravitation, volume 27, pages 657-667.

[7]: R. Aldrovandi, G. E. A. Matsas, S. F. Novaes, D. Spehler, (1994), " Fermion Helicity Flip in Weak Gravitational Fields", arXiv:gr-qc/9404018v1

[8]: Soumitra SenGupta, Aninda Sinha, (2001), " Fermion helicity flip by parity violating torsion", arXiv:hepth/0102073v2.

[9]: Stephane H Maes, (2020), "Gravity Induced Anomalies Smearing in Standard Model so that Protons May Never Decay, Except in Black Holes ", viXra:2006.0128v1, https://shmaesphysics.wordpress.com/2020/06/12/protonsmay-never-decay-except-in-black-holes/, June 13, 2020.

[10]: https://en.wikipedia.org/wiki/Neutrino

[11]: M.C. Gonzalez-Garcia and M. Yokoyama, (2019), "14. Neutrino Masses, Mixing, and Oscillations", in M. Tanabashi et al. (Particle Data Group), Phys. Rev. D 98, 030001 (2018) and (2019) update. 